\title{
Simulación Clínica, Realidad Virtual Háptica(RVH): Herramienta docente para la enseñanza en estomatología
}

\author{
Clinical Simulation, Virtual Haptic Reality (VHR): Teaching tool for dental education.
}

La Simulación Clínica, es una herramienta didáctica que busca el logro de competencias en un entorno lo más cercano al real, y siempre se ha utilizado en mayor o menor medida; históricamente se han empleado dientes naturales para las prácticas preclínicas, y en condiciones no siempre adecuadas, así como modelos de yeso o dientes de plástico que ofrece el mercado; se ha documentado sobre los riesgos que implica el uso de estas formas tradicionales, en términos legales, de bioseguridad, y las limitaciones en cuanto a fidelidad y realismo, todas estas desventajas en conjunto generan una brecha entre la teoría y la práctica del estudiante.

El desarrollo de la tecnología ha logrado implementar herramientas didácticas en muchas disciplinas, se han logrado reproducir entornos virtuales que proveen herramientas nunca antes vistas, que crean un entorno seguro y con alto grado de realismo. En la actualidad estas herramientas ya están disponibles para todos los niveles educativos, y la velocidad con la que se esta dando ha generado que vivamos una transición generacional compleja.

El aprendizaje de esta generación esta expuesta a esta tecnología desde niveles básicos de formación, tal es así que nuestros alumnos que ingresan al pregrado, ya son nativos digitales, y los docentes se ven obligados a ser inmigrantes digitales, esta situación obliga a la denominada "alfabetización digital" que constituye un desafío y que puede costar una serie de dificultades para el grupo docente y para la institución, pues la simple inserción de las herramientas tecnológicas no garantizan su eficacia educativa.

En este contexto, el proceso de implementación de la tecnología debe ir acompañado de la inmersión de los docentes en la nueva estrategia didáctica que implica el uso de la tecnología, en especial desde periodos tempranos de formación, así como en fases preclínicas, con el objetivo lograr una mejor transición entre la teoría y la práctica y reducir el estrés en el estudiante en formación, por ende, lograr mejores resultados de aprendizaje, en un entorno más seguro para el estudiante y consecuentemente para el paciente.

La Facultad de Estomatología Roberto Beltrán de la Universidad Peruana Cayetano Heredia ha apostado por la implementación tecnológica de la mano con la capacitación docente, inaugurándose, a finales del año pasado, el centro de simulación virtual en estomatología más grande de Latinoamérica, como parte de un proyecto pensado a largo plazo, donde la tecnología esta presente, no para reemplazar, si no para complementar el proceso de aprendizaje.

La adquisición de los equipos de simulación virtual háptica, Simodont (MOOG), significa un salto tecnológico importante para nuestra institución, y el equipo docente en formación entiende que la estrategia de simulación tiene múltiples utilidades que hacen del proceso de aprendizaje una experiencia mucho más eficiente, segura y amigable con el medio ambiente, con el objetivo de desarrollar una de las habilidades que todo odontólogo debe 
adquirir desde un inicio de la carrera, la destreza manual, integrada a la adquisición del conocimiento secuencial, desde los niveles básicos hasta los más avanzados en postgrado,

Así mismo, esta tecnología nos permite una evaluación objetiva y en tiempo real de cada procedimiento, permitiendo al estudiante corregir su práctica de inmediato, esta información nos permitirá explorar la evolución del proceso de adquisición de la habilidad en diferentes niveles y perspectivas didácticas y de investigación, así como tener una retroalimentación de los procesos adoptados, necesarios para su mejora continua.

El equipo de realidad virtual háptico, Simodont, el cual ha sido desarrollado por Moog y el Centro Académico de Odontología de Ámsterdam (ACTA), brinda la capacidad háptica (término que viene del griego "haptos" que significa "Tocar"), incorporando a la experiencia la característica de poder tocar lo que se ve en 3D. Desde el punto de vista didáctico, se ha demostrado que, cuando se agrega un sentido más a la experiencia de aprendizaje, la capacidad de retención de eleva exponencialmente.

Asimismo, al ser una estrategia integrada en la que se aprende en un contexto de alto realismo, se genera esa dosis de emoción, característica ideal en todo proceso óptimo de aprender "haciendo" en un entorno seguro, recordemos lo que Confucio decía: "Cuando oigo, olvido, cuando veo, recuerdo; Pero cuando hago, entiendo".

\section{Edgar Quenta Silva ${ }^{1, a, b}$}

